\title{
Depresión en el adulto mayor intervenido quirúrgicamente
}

\section{Depression in the surgically intervened elderly adult}

\author{
Javier Varela-Montes ${ }^{*}$, Celina García-Guzmán² y Héctor Cobos-Aguilar ${ }^{3}$ \\ ${ }^{1}$ Departamento de Coordinación de Educación e Investigación en Salud, Hospital General de Zona No. 4, Instituto Mexicano del Seguro Social \\ (IMSS), Zamora, Michoacán; ${ }^{2}$ Departamento de Cirugía, Hospital Rural No. 30, IMSS, Ario de Rosales, Michoacán; ${ }^{3}$ Departamento de Vicerrectoría \\ en Ciencias de la Salud, Universidad de Monterrey, San Pedro Garza García, Nuevo León. México
}

\section{Resumen}

Objetivo: Determinar el grado de depresión en el adulto mayor tras una cirugía y su relación con el tiempo anestésico. Método: Estudio observacional, comparativo, prospectivo y longitudinal. Se incluyeron 73 adultos mayores de 60 años programados para diferentes cirugías. Se evaluó el grado de depresión antes y después de la cirugía con la Escala de Depresión Geriátrica de Yasavage versión corta. Se clasificó según la puntuación: sin depresión (0-5), depresión leve (6-9) y depresión establecida (10-15). Se relacionó la depresión con el tiempo anestésico. El tamaño muestral se calculó para proporciones. Se utilizó estadística descriptiva y prueba $\chi^{2}(p<0.05)$. Resultados: En la primera evaluación se observaron 47 (64\%) pacientes sin depresión, 21 (29\%) con depresión leve y 5 (7\%) con depresión establecida. En la segunda evaluación se encontraron 44 (60\%) pacientes sin depresión, 21 (29\%) con depresión leve y 8 (11\%) con depresión establecida. Para la relación entre depresión y tiempo anestésico, $\chi^{2}=0.81$. Conclusiones: No se encontró relación entre la depresión y el tiempo anestésico en adultos mayores intervenidos quirúrgicamente.

Palabras clave: Adulto. Anestesia. Depresión. Envejecimiento. Espinal.

\begin{abstract}
Objective: To determine the degree of depression in elderly adults after surgery and its relation with the duration of anesthesia. Method: We conducted an observational, comparative, prospective and longitudinal study. We included 73 elderly adults aged 60 scheduled for different surgical procedures. Their degree of depression was evaluated prior to and after the surgery with the short version of the Yasavage Geriatric Depression Scale. They were classified according to the score: no depression (0-5), mild depression (6-9) and established depression (10-15). The relation of depression with anesthesia duration was determined. The sample size was calculated for proportions. Descriptive statistics were used as well as $\chi^{2}(p<0.05)$. Results: In the first evaluation 47 patients (64\%) were not depressed, 21 (29\%) had mild depression and 5 (7\%) had established depression. In the second evaluation, we found that 44 patients (60\%) were not depressed, 21 (29\%) had mild depression and 8 (11\%) had established depression. The relation between depression and anesthesia duration was $\chi^{2}=0.81$. Conclusions: We did not establish a relation between depression and anesthesia duration in surgically intervened elderly adults.
\end{abstract}

Key words: Adult. Anesthesia. Depression. Aging. Spinal. 


\section{Introducción}

La pirámide poblacional en México se ha invertido en los últimos lustros debido a factores como el control de la natalidad y las infecciones; por tal motivo, la esperanza de vida se ha prolongado y cada vez hay más ancianos en nuestro país. Se calcula que para el año 2050 una cuarta parte de los mexicanos tendrá más de 60 años. La demanda en los servicios médicos y los altos costos serán un gran reto para atender a este grupo de edad y sus enfermedades asociadas, como diabetes, enfermedades cardiovasculares, trastornos neuropsiquiátricos (depresión y ansiedad) y enfermedades neurodegenerativas que cursan con pérdida de las capacidades cognitivas, funcionales y emocionales, que limitan las actividades diarias y la vida independiente.

El envejecimiento se asocia a pérdida de la reserva neuronal, disminución del flujo sanguíneo cerebral, lenta conducción nerviosa, mala coordinación psicomotriz, alteraciones en la capacidad para resolver problemas complejos y pérdida de la memoria reciente. A esta pérdida progresiva de las funciones mentales superiores que se presenta durante el proceso de envejecimiento se asocia la depresión, que para el año 2020, según la Organización Mundial de la Salud, será la segunda causa de morbimortalidad en adultos mayores hospitalizados por causas médicas o quirúrgicas con riesgo de complicación, que se incrementa en estas últimas ${ }^{1-3}$.

La depresión es un trastorno neuropsiquiátrico de alta prevalencia en pacientes hospitalizados en los servicios tanto médicos como quirúrgicos; su prevalencia es del $16-43 \%$ en los pacientes que ingresan por enfermedades agudas.

La depresión se caracteriza por síntomas afectivos, como tristeza, anhedonia o disforia, y por síntomas somáticos, como alteraciones del sueño, del apetito o neurovegetativas (palpitaciones, dolor precordial, disnea, hiperventilación, sudoración, náuseas, escalofríos). Puede haber algunos síntomas psicológicos, como sensación de muerte, pérdida de autocontrol y despersonalización.

El paciente pierde el interés por vivir, muestra incapacidad para realizar actividades y presenta alteraciones del sueño, pérdida o ganancia de peso, insomnio o hipersomnia, agitación, enlentecimiento psicomotor, fatiga o pérdida de energía, sentimiento de ser inútil o de culpa, alteraciones en la capacidad de pensar o concentrarse, pensamientos de muerte e ideación suicida, que interfieren en su calidad de vida. En resumen, es un trastorno heterogéneo de curso indeterminado y respuesta variable al tratamiento.

Las personas que padecen alguna enfermedad crónica o discapacitante, o que son candidatas para realizar alguna intervención quirúrgica, son propensas a sufrir depresión, por lo que identificar la comorbilidad es de gran importancia por el impacto en su calidad de vida ${ }^{4-10}$.

La atención del adulto mayor requiere una evaluación integral que considere las actividades de la vida diaria y su funcionalidad, la dinámica familiar, las condiciones socioeconómicas y el estado neuropsicológico ${ }^{11}$.

Es aconsejable que los procedimientos quirúrgicos en estos pacientes sean programados y de mínima invasión, pues se trata de evitar una cirugía de urgencia por las complicaciones graves, físicas y mentales, a las que están asociadas. La disfunción cognitiva, la edad avanzada, la precariedad social y la incapacidad del individuo están relacionadas con una menor probabilidad de recuperación y con un mayor riesgo de muerte ${ }^{12-16}$.

Para evaluar si el adulto mayor con algún tipo de cirugía programada presenta disfunción del estado mental, específicamente depresión, se cuenta con varias pruebas diagnósticas. Una de ellas es la Escala de Depresión Geriátrica de Yesavage, versión corta, de 15 ítems, que es una herramienta útil, sencilla de utilizar y con una sensibilidad del $97 \%$ y una especificidad del 85\% para detectar depresión. La respuesta a cada pregunta es dicotómica: afirmación (sí) o negación (no). Diez preguntas están elaboradas para ser respondidas de manera afirmativa (sí) y cinco de manera negativa (no). Si las respuestas de los pacientes coinciden con las respuestas afirmativas o negativas, se asigna una puntuación de 1 . Al final se suman los puntos y de acuerdo con estos se emite el punto de corte: $0-5$ puntos, sin depresión; 6-9 puntos, depresión leve; 10-15 puntos, depresión establecida.

El presente trabajo tiene como objetivo determinar el grado de depresión en adultos mayores tras someterse a una cirugía y su relación con el tiempo anestésico.

\section{Método}

Se realizó un estudio observacional, comparativo, prospectivo y longitudinal en el servicio de cirugía general de un hospital general de zona en un período 
de 6 meses. Se incluyeron adultos de 60 años y más de edad, de ambos sexos, que sabían leer y escribir, programados de manera electiva para cirugía abdominal, urológica, ortopédica u oncológica, o para amputaciones, con anestesia regional.

Se excluyeron pacientes que fueron operados de urgencia y aquellos sin valoración quirúrgica en el expediente o que no estuvieron en la lista de programación quirúrgica, con antecedentes de depresión o que hubieran tomado antidepresivos. Se eliminaron los pacientes que fueron operados electivamente pero que egresaron del quirófano posintubados porque recibieron anestesia general.

Los datos generales se tomaron del expediente clínico. Se evalúo el grado de depresión mediante la Escala de Depresión Geriátrica de Yasavage, versión corta (15 preguntas) (Tabla 1).

La encuesta se aplicó por personal médico capacitado en el servicio de ingreso del paciente. El procedimiento fue el siguiente: el entrevistador se colocó frente al paciente y leyó cada pregunta con voz fuerte, lento y claro; el paciente, una vez que comprendió la pregunta, contestó verbalmente con afirmación (sí) o negación (no). Las preguntas 2-4, 6, 8-10, 12 y 14-15 son para responderlas afirmativamente (sí), y las preguntas 1,5 , 7, 11 y 13 negativamente (no). Cuando el paciente coincidió con las respuestas afirmativas o negativas se asignó 1 punto. El entrevistador sumó todas las respuestas que se calificaron con puntuación de 1 , y los puntos de corte fueron los siguientes: 0-5, sin depresión; 6-9, depresión leve; 10-15, depresión establecida.

La evaluación se aplicó en dos tiempos: antes de la cirugía en el servicio donde estuvieron hospitalizados, con el paciente tranquilo, sin dolor y acostado, y después de la cirugía en la sala de recuperación, con el paciente tranquilo, sin dolor, acostado, con buena comunicación oral y con movimientos de las extremidades inferiores.

El tiempo anestésico se midió en minutos con un cronómetro convencional por la misma persona que realizó la encuesta, considerando desde el momento en que el anestesiólogo inició el bloqueo regional hasta que el paciente movilizó las extremidades inferiores en la sala de recuperación.

El tamaño de la muestra se calculó con el programa Epi Info versión 3, para variables cualitativas para estimar proporciones (n: 73), en una población finita con un intervalo de confianza del $95 \%$ y una significación establecida en $p=0.53$. La selección de pacientes se realizó por conveniencia, respetando los criterios de selección, el anonimato y la confidencialidad.
Tabla 1. Escala de Depresión Geriátrica de Yesavage (versión corta)

\begin{tabular}{llcc}
\hline & & Sí & No \\
\hline 1 & ¿Está satisfecho/a con su vida? & 0 & 1 \\
2 & ¿Ha renunciado a muchas actividades? & 1 & 0 \\
3 & ¿Siente que su vida está vacía? & 1 & 0 \\
4 & ¿Se encuentra a menudo aburrido/a? & 1 & 0 \\
5 & ¿Tiene a menudo buen ánimo? & 0 & 1 \\
6 & ¿Teme que algo malo le pase? & 1 & 0 \\
7 & ¿Se siente feliz muchas veces? & 0 & 1 \\
8 & ¿Se siente a menudo abandonado/a? & 1 & 0 \\
9 & ¿Prefiere quedarse en casa que salir? & 1 & 0 \\
10 & ¿Cree tener más problemas de memoria que la & 1 & 0 \\
& mayoría de la gente? & & \\
11 & ¿Piensa que es maravilloso vivir? & 0 & 1 \\
12 & ¿Le cuesta trabajo iniciar nuevos proyectos? & 1 & 0 \\
13 & ¿Se siente lleno/a de energía? & 0 & 1 \\
14 & ¿Siente que su situación es desesperada? & 1 & 0 \\
15 & ¿Cree que mucha gente está mejor que usted? & 1 & 0
\end{tabular}

Puntuación total

Interpretación:

0 a 5: normal.

6 a 9: depresión leve.

10 o más: depresión establecida.

Se utilizó estadística descriptiva para el análisis de los datos como media, mediana, moda y medidas de dispersión, y la prueba $\chi^{2}$ para relacionar las variables cualitativas, mediante el programa estadístico SPSS versión 23.

\section{Resultados}

Se recabaron 87 pacientes, de los que 73 ingresaron al estudio por cumplir los criterios de inclusión. Se excluyeron 12 pacientes por cirugía de urgencia y se eliminaron 2 posintubados porque recibieron anestesia general en el transoperatorio. De los que ingresaron al estudio, 47 (64\%) eran hombres y 26 (36\%) eran mujeres, la edad promedio fue de 71 años (desviación estándar: 8.6). En escolaridad 17 (23\%) fueron analfabetas, 24 (33\%) con primaria incompleta, $13(18 \%)$ primaria completa, secundaria incompleta $3(4 \%)$, secundaria completa $7(9 \%)$, bachillerato incompleto $1(1 \%)$, bachillerato completo $4(6 \%)$, profesional 4 (6\%). El servicio de cirugía general fue el 
que más intervenciones quirúrgicas realizó, con 23 (32\%) casos. El diagnóstico preoperatorio más frecuente fue la resección transuretral prostática en 15 (21\%). Los resultados de la evaluación del grado de depresión antes de la cirugía mostraron 47 (64\%) pacientes sin depresión, 21 (29\%) con depresión leve y $5(7 \%)$ con depresión establecida. Después de la cirugía, 44 (60\%) pacientes no tuvieron depresión, 21 (29\%) mostraron depresión leve y 8 (11\%) depresión establecida. Los hombres fueron los que más se deprimieron antes y después de la cirugía. El promedio del tiempo anestésico fue de 156 minutos (desviación estándar: 61).

El resultado entre la depresión y el tiempo anestésico fue de $\chi^{2}=0.81$ (no significativo).

\section{Discusión}

La prevalencia de depresión, con esta escala, en esta muestra de pacientes adultos mayores programados quirúrgicamente de manera electiva, antes de realizar la cirugía fue del $36 \%$ y después de la cirugía se incrementó un $4 \%$. En los últimos reportes que hay en la literatura se menciona que en nuestro país la prevalencia de depresión en el adulto mayor se encuentra entre el $26 \%$ y el $66 \%$, aunque la mayoría de estos estudios fueron realizados en pacientes hospitalizados por diversas causas y solo pocos reportes incluyeron causas quirúrgicas ${ }^{3}$. Nuestro resultado es diferente al que se reporta en otros países, por ejemplo Brasil, con una prevalencia de depresión del $22 \%$ en pacientes sometidos a cirugía electiva, mientras que en Europa es menor ${ }^{17-19}, y$ tal vez estas cifras más bajas se deben al método diagnóstico y a los criterios utilizados, porque cuando se utilizan los criterios de la Clasificación Internacional de Enfermedades, $10 .^{\text {a }}$ edición (CIE-10) o los del Manual Diagnóstico y Estadístico de los Trastornos Mentales, $5 .^{\text {a }}$ edición (DSM-5) el resultado de la prevalencia de depresión es menor comparado con los resultados obtenidos con pruebas de tamizaje como la Escala de Depresión Geriátrica, utilizada en este reporte, o cuando se utilizan otros cuestionarios ${ }^{20}$.

Por otro lado, los hallazgos en relación con la prostatectomía y el nivel educativo coinciden con lo reportado en la literatura, porque después de la prostatectomía, los pacientes, por falta de conocimiento de su recuperación o de autocuidado posoperatorio, presentan depresión y requieren apoyo psicológico para adaptarse a su nueva vida, enfrentar su enfermedad y mejorar su calidad de vida.
El hospital donde se realizó el estudio, de mayor complejidad que los hospitales de su tipo, no ofrece apoyo psicológico, ya que no cuenta con servicio de psicología, que es imprescindible para el éxito en todo el proceso, y algunos estudios que describen la participación del psicólogo como parte fundamental del equipo multidisciplinario reportan resultados favorables. Es necesario que los hospitales cuenten con el apoyo de un psicólogo para enfrentar de mejor manera las necesidades, cada vez más apremiantes, de los adultos mayores hospitalizados. Un estudio encontró que en los adultos de 70 a 84 años de edad con depresión aumenta el riesgo de morir en 3 años comparados con los que no la presentan ${ }^{21}$, por lo que es necesario diagnosticarla tempranamente en este grupo de pacientes e iniciar tratamiento farmacológico y no farmacológico tan pronto como sea posible.

Nuestro estudio presenta como limitación que el instrumento de cribado utilizado identifica a los pacientes en riesgo de depresión, y el diagnóstico definitivo se realiza con una entrevista psiquiátrica, no llevada a cabo en este reporte.

\section{Conclusiones}

La prevalencia de depresión en los adultos mayores que se programan para cirugía electiva, en nuestra población estudiada, antes de la intervención quirúrgica fue del $36 \%$ y después de la cirugía se incrementó un $4 \%$.

No existe relación entre el tiempo anestésico y la depresión en los adultos mayores intervenidos quirúrgicamente.

Los adultos mayores programados para cirugía deben contar con una evaluación del estado mental que permita diagnosticar la depresión y proporcionar tratamiento psicológico o farmacológico oportunamente; de esta manera se incide en la morbimortalidad y se mejora la calidad de vida de estos pacientes.

\section{Agradecimientos}

Los autores agradecen a los pacientes que participaron en el presente estudio y a todas las personas que nos dieron su apoyo moral para la culminación de este proyecto.

\section{Conflicto de intereses}

Los autores declaran que no existe ningún conflicto de intereses. 


\section{Responsabilidades éticas}

Protección de personas y animales. Los autores declaran que para esta investigación no se han realizado experimentos en seres humanos ni en animales.

Confidencialidad de los datos. Los autores declaran que han seguido los protocolos de su centro de trabajo sobre la publicación de datos de pacientes.

Derecho a la privacidad y consentimiento informado. Los autores declaran que en este artículo no aparecen datos de pacientes.

\section{Bibliografía}

1. Chávez-Ramírez DR, Zegbe JA, Sánchez-Morales FM, Castañeda-Iñiguez MS. Depresión en adultos mayores atendidos en instituciones públicas de salud en Zacatecas. Rev Educ Desarro. 2014;3:73-8.

2. Muñiz-Chavelas $M$, Núñez-Trenado LA, López-Flores C, Baquero MM García-Álvarez J, Pérez-García R. Abdomen agudo quirúrgico en el paciente geriátrico. Rev Hosp Jrz. 2007;74:174-85.

3. Hernández-Zamora ZE, Vásquez-Espinosa JM, Ramo-Mendoza A. Depresión en adultos mayores hospitalizados. Una propuesta integral de intervención del psicólogo. Rev Electr Med Salud Soc. 2012;3:1-27.

4. Sotelo-Alonso I, Rojas-Soto JE, Sánchez-Arenas C, Irigoyen-Coria A. La depresión en el adulto mayor: una perspectiva clínica y epidemiológica desde el primer nivel de atención. Arch Med Fam. 2012;14:5-13.

5. Flores-Castro M, Arias-Merino ED. Depresión en el adulto mayor. En: Ortiz GG, editor. El envejecimiento: aspectos básicos, clínicos y sociales. México; 2003. p. 277-301.

6. Martínez-Mendoza JA, Martínez-Ordaz VA, Esquivel-Molina CG, Velasco-Rodríguez VM. Prevalencia de depresión y factores de riesgo en el adulto mayor hospitalizado. Rev Med Inst Mex Seguro Soc. 2007;45:21-8.
7. Varela-Pinedo L, Cháez-Jimeno $\mathrm{H}$, Gálvez-Cano $\mathrm{M}$, Mendez-Silva $\mathrm{F}$. Funcionalidad en el adulto mayor previa a su hospitalización a nivel nacional. Rev Med Herediana. 2005;16:165-71.

8. Castro-Lizárraga M, Ramírez-Zamora S, Aguilar-Morales LV, Díaz-de Anda VM. Factores de riesgo asociados a la depresión del adulto mayor. Rev Neurol Neurocir Psiquiat. 2006;39:132-7.

9. Aguilar-Navarro S, Ávila-Funes JA. La depresión: particularidades clínicas y consecuencias en el adulto mayor. Gac Med Mex. 2007;143:141-8.

10. Grau-Martín A, Suñer-Soler R, Abili-Picart P, Comas-Casanovas P. Niveles de ansiedad y depresión en enfermos hospitalizados y su relación con la gravedad de la enfermedad. Med Clin (Barc). 2003;120:370-5.

11. Medina-Chávez JH, Torres-Arreola LP, Cortés-González RM, Durán-Gómez V, Martínez-Hernández F, Esquivel-Romero G. Guía de práctica clínica. Valoración geriátrica integral. Rev Inst Mex Seguro Soc. 2011;49:669-84.

12. Csendes JA, Lembach JH, Molina C, Inostroza LG, Köbrich SS. Cirugía en mayores de 80 años: evolución clínica y costos asociados. Rev Chil Cir. 2010;62:564-9.

13. Campo-Arias A, Urruchurtu-Mendoza Y, Solano-Morales T, Vergara-Pino AJ, Cogollo Z. Consistencia interna, estructura factorial y confiabilidad del constructo de la Escala de Yesavage para depresión geriátrica (GDS-15) en Cartagena (Colombia). Salud Uninort. 2008;24:1-9.

14. Torrens-Darder M. Depresión en geriatría: diagnóstico diferencial y tratamiento. Rev Psiq Fac Med Barna. 2001;28:239-46.

15. Fernández-Santos CM. Depresión el anciano. Med Gen. 2002;40:28-31.

16. Rosas-Flores MA, Cossío-Zazueta A, Henestrosa-Peña K, Flores-Escartín M, Cal y Mayor-Turnbull I, Meza-Vudoyra M, et al. Prevalencia de depresión en pacientes con pie diabético. Rev Mex Angiol. 2006;34:107-11.

17. Ferreira-da Mata LR, Campos-Carvalho EC, Gontijo-Gomes CR, DaSilva AC, DaGraça-Pereira M. La autoeficacia y la morbilidad psicológica en el postoperatorio de la prostatectomía radical. Rev Latinoam Enfermagem. 2015;23:806-13.

18. Avelino-DosSantos M, Aparecida-Rossi L, Paiva L, Spadoti-Dantas RA Alcalá-Pompeo D, Bomfim-Machado EC. Medida da ansiedade e depressao em pacientes no pre-operatorio de cirugías electivas. Rev Eletr Enferm. 2012;14:222-7.

19. Korfage IJ, Essink-Bot ML, Janssens ACJW, Schröder FH, DeKoning HJ. Anxiety and depression after prostate cancer diagnosis and treatment: 5-year follow-up. Br J Cancer. 2006;94:1093-8.

20. Calderón MD. Epidemiología de la depresión en el adulto mayor. Rev Med Hered. 2018:29:182-91.

21. Rodda J, Walker Z, Carter J. Depression in older adults. BMJ. 2011;343:683-7. 